\title{
Soft and Isotropic Phonons in $\mathrm{PrFeAsO}_{1-y}$
}

\author{
T. Fukuda, ${ }^{1,2,3}$ A.Q.R. Baron, ${ }^{1,4,3}$ H. Nakamura, ${ }^{5,3}$ S. Shamoto, ${ }^{6,3}$ M. Ishikado, ${ }^{6,3}$ \\ M. Machida, ${ }^{5,3}$ H. Uchiyama,,${ }^{1,4}$ A. Iyo, ${ }^{7,3}$ H. Kito, ${ }^{7,3}$ J. Mizuki, ${ }^{2,3}$ M. Arai, ${ }^{8}$ and H. Eisaki ${ }^{7,3}$ \\ ${ }^{1}$ Materials Dynamics Laboratory, RIKEN SPring-8 Center, Sayo, Hyogo 679-5148, Japan \\ ${ }^{2}$ Quantum Beam Science Directorate, JAEA SPring-8, Sayo, Hyogo 679-5148, Japan \\ ${ }^{3}$ JST, Transformative Research-Project on Iron Pnictides (TRIP), Chiyoda, Tokyo, 102-0075, Japan \\ ${ }^{4}$ Research and Utilization Division, JASRI SPring-8, Sayo, Hyogo 679-5198, Japan \\ ${ }^{5}$ JST, CREST and the Center for Computational Science and e-Systems, JAEA, Taito, Tokyo, 110-0015, Japan \\ ${ }^{6}$ Quantum Beam Science Directorate, JAEA, Naka, Ibaraki 319-1195, Japan \\ ${ }^{7}$ Nanoelectronics Research Institute (NeRI), AIST, Tsukuba, Ibaraki, 305-8568, Japan \\ ${ }^{8}$ J-PARC Center, JAEA, Naka, Ibaraki, 319-1195, Japan
}

(Dated: November 25, 2018)

\begin{abstract}
Phonons in single crystals of $\mathrm{PrFeAsO}_{1-y}$ are investigated using high-resolution inelastic x-ray scattering and ab-initio pseudo-potential calculations. Extensive measurements of several samples ( $y \sim 0,0.1$ and 0.3$)$ at temperatures spanning the magnetic ordering temperature $\left(T_{N} \sim 145 \mathrm{~K}\right.$ for $y \sim 0)$ and the superconducting transition temperature $\left(T_{c}=36 \mathrm{~K}\right.$ for $y \sim 0.1$ and $T_{c}=45 \mathrm{~K}$ for $y \sim 0.3$ ) show that there are some changes in phonon spectra with temperature and/or doping. We compare our measurements with several ab initio pseudo-potential models (nonmagnetic tetragonal, oxygen-deficient $\mathrm{O}_{7 / 8}$ supercell, magnetic orthorhombic, and magnetic tetragonal) and find that the experimentally observed changes are much smaller than the differences between the experimental data and the calculations. Agreement is improved if magnetism is included in the calculations via the local spin density approximation, as the Fe atomic motions parallel to the ferromagnetic ordering direction are softened. However, the antiferromagnetically polarized modes remain hard, and in disagreement with the experimental data. In fact, given the increasing evidence for anisotropy in the iron pnictide materials, the phonon response is surprisingly isotropic. We consider several modifications of the $a b$ initio calculations to improve the agreement with the experimental data. Improved agreement is found by setting the matrix to zero (clipping the bond) between nearest-neighbor antiferromagnetically aligned Fe atoms in the magnetic calculation, or by softening only the in-plane nearest-neighbor Fe-As force constant in the nonmagnetic calculation. We discuss these results in the context of other measurements, especially of phonons, for several FeAs systems. Fluctuating magnetism may be a partial explanation for the failure of the calculations, but seems incomplete in the face of the similarity of the measured phonon response in all the systems investigated here including those known to have static magnetism.
\end{abstract}

PACS numbers: 74.25.Kc, 74.70.Xa, 78.70.Ck

\section{INTRODUCTION}

Since the discovery of superconductivity in $\mathrm{LaFeAs}(\mathrm{O}, \mathrm{F})$ at $26 \mathrm{~K}$ (Ref. 1), iron-arsenides and related compounds have been the subject of enormous scientific attention. These compounds have a layered structure consisting of iron and pnictide/chalcogenide atoms ("Fe-As layer") with each iron atom surrounded by a tetrahedron of pnictide/chalcogenide atoms (c.f. Fig. (1). The iron-based superconductors have been classified into, mainly, four groups according to the kind of buffer between the Fe-As layers: $R \mathrm{FeAs} X(R$ : rare earth, $X=\mathrm{O}$ or $\mathrm{F}$ ) with $\mathrm{ZrCuSiAs}$ type structure ("1111") $1, \underline{1,-7}, R \mathrm{Fe}_{2} \mathrm{As}_{2}(R=\mathrm{Ba}, \mathrm{Sr}, \mathrm{Ca})$ with $\mathrm{ThCr}_{2} \mathrm{Si}_{2}$ type structure ("122") $)^{8.9}, A \mathrm{FeAs}(A=\mathrm{Li}, \mathrm{Na})$ with $\mathrm{Cu}_{2} \mathrm{Sb}$ type tetragonal structure (" $\left.111 "\right) 10,11$, and $\alpha-\mathrm{Fe} C h(C h=\mathrm{S}, \mathrm{Se}, \mathrm{Te})$ with $\alpha$-PbO type structure ("11") 12,13 . Doping electron or hole carriers into the Fe-As layers, or application of pressure (external or chemical) results in superconductivity at low temperature. Some of the "1111" type materials show superconductivity above $50 \mathrm{~K}$ (Refs. 5), which is the highest observed superconducting transition temperature $\left(T_{c}\right)$ outside of the copper oxide family of high- $T_{c}$ superconductors. Recently more complex compounds having a buffer layer of perovskite structure have been synthesized pursuing a higher $T_{c}(\operatorname{Refs} \underline{\underline{14}} \underline{\underline{19}})$. However, the highest $T_{c}$ is still achieved in a "1111" material.

It has been suggested by many groups that the physical properties of iron-arsenic compounds are very sensitive to the crystal structure, especially to the pnictide/chalcogenide height above the Fe plane and/or the angle of the As-Fe-As bonds $20-25$. This is further emphasized by the possibility to dramatically affect the superconducting properties of some material by applying pressure, with $T_{c}$ increased in $\operatorname{LaFeAs}(\mathrm{O}, \mathrm{F})$ from 26 to $43 \mathrm{~K}$ under an applied pressure of $4 \mathrm{GPa}$ (Ref. 26), and in $\mathrm{Fe}_{1.01}$ Se from 8.5 to $36.7 \mathrm{~K}$ under a pressure of $8.9 \mathrm{GPa}$ (Ref. 27). This sensitivity suggests the possibility of large coupling between lattice motion and electronic or, perhaps, magnetic structure. Meanwhile, early first principles calculations of phonon properties suggested that the superconductivity in ironarsenic compounds is not phonon mediated $\underline{28} \underline{-30}$. How- 


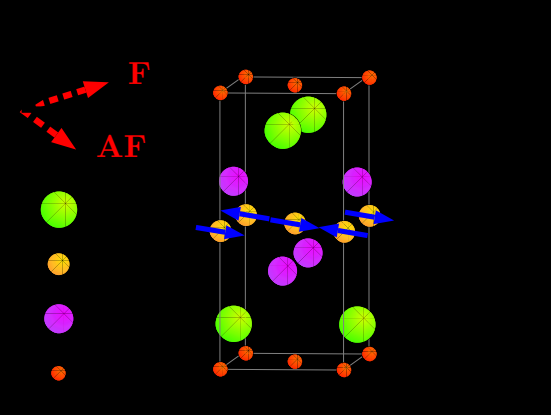

FIG. 1: (Color online) Crystal structure of ReFeAsO (Re: rare earth). The arrows on Fe atoms show the magnetically ordered pattern observed for $T<T_{N}$ (Refs. 1 44). Dashed (red) arrows indicate the direction of ferromagnetic (F) [antiferromagnetic (AF)] alignment of iron spins in the plane.

ever, later calculations 31.32 demonstrated the strong dependence of the iron magnetic moment on the pnictide/chalcogenide atomic position, suggesting some interplay between phonons and magnetism, while others have noted increased electron-phonon in magnetic calculations by factors of $\sim 2$ (Ref. 33) to $\sim 10$ (Ref. 34).

Experimentally phonons have been measured using various methods. Inelastic neutron and/or x-ray scattering (INS/IXS) is a powerful tool to investigate phonon structures, and many measurements have been already reported $35-51$. Many of these reported small changes with doping and/or temperature. The experimental phonon data are compared to first principles calculations, and the introduction of magnetic effect is thought to be important $\underline{45-51}$. We will discuss them in detail later. Here we note that with the exception of Refs. 37 and 49 all single crystal work has focused on the "122" samples, despite their lower $T_{c}$, because the "1111" materials remain relatively difficult to grow.

In the present paper, we report phonon dispersion measurements along various symmetry directions measured on single crystals of $\mathrm{PrFeAsO}_{1-y}$ using IXS. This is a "1111" compound that shows relatively high $T_{c}$ up to $45 \mathrm{~K}$ for the samples discussed here and $49 \mathrm{~K}$ for the Pr family. We also perform several first principles calculations, and compare them carefully with the experimental data. Our detailed observation confirmed the isotropy of phonon dispersion even in the antiferromagnetically ordered phase compared to the first principles calculation considering the magnetic effect. Though some similar observations, calculations, and the comparisons have been already discussed for other iron-arsenide compounds ${ }^{35-51}$, the similarity of our results to other works ensures that the present work contains the general features of iron-arsenides.

We then consider several modifications of the first principles calculations to better interpret our results. Two modified models are found to have good agreement with the experimental data. Interestingly, one is the model that adds additional in-plane anisotropy, while the other preserves the isotropy of our nonmagnetic first principles
TABLE I: PrFeAsO ${ }_{1-y}$ samples. The thickness of the samples was a few tens $\mu \mathrm{m}$. The sample 'doped-1' was also used in a previous study (Ref. 37).

\begin{tabular}{cccccc}
\hline \hline name & $y$ & $T_{s}[\mathrm{~K}]$ & $T_{N}[\mathrm{~K}]$ & $T_{c}[\mathrm{~K}]$ & typical size $[\mu \mathrm{m}]$ \\
\hline parent & 0.0 & $\sim 149$ & $\sim 139$ & - & $\sim 500$ \\
doped-1 & $\sim 0.1$ & - & - & 36 & $\sim 100$ \\
doped-2 & $\sim 0.3$ & - & - & 45 & $\sim 300$ \\
\hline \hline
\end{tabular}

calculation.

This paper is organized as follows. The details of samples investigated, IXS measurements, and first principles calculations are provided in Sec. II The experimental results and the comparison with calculations are given in Sec. III. Several modification model of first principles calculations are detailed in Sec. IV Section V is dedicated to the discussion of the results and a summary is given in Sec. VI.

\section{EXPERIMENTS AND CALCULATIONS}

\section{A. Samples}

The investigated $\mathrm{PrFeAsO}_{1-y}$ single crystals are summarized in Table I] They were prepared using highpressure growth as described in Ref. 52. The first samples were relatively small $\sim 0.1 \times 0.15 \times 0.02 \mathrm{~mm}^{3}$, but with improvements in growth techniques, they became comfortably large, $\sim 0.5 \mathrm{~mm}$ in the $a b$ plane. Sample thickness varied from about 0.02 to $0.05 \mathrm{~mm}$. X-ray diffraction on a four-circle diffractometer was used to verify that all samples were single grains, with, typically, a mosaic spread of about $1^{\circ}$. The $T_{c}$ 's of the studied superconducting samples were found to be 36 and $45 \mathrm{~K}$ by measuring magnetic susceptibility using a superconducting quantum interference device (SQUID) magnetometer. The electrical resistivity in the parent sample showed an abrupt change at $\sim 149 \mathrm{~K}$ and the derivative had a maximum at $\sim 139 \mathrm{~K}$ (Fig 2). According to the previous transport measurements in iron pnictides ${ }^{53,54}$, these temperatures correspond to the structural and magnetic transition, respectively. These temperatures are slightly higher than the values given in Refs. 3 and 55, possibly because we are more nearly at the precise stoichiometric composition. There is a small anomaly in $\rho$ around 12 $\mathrm{K}$ where the Pr spins order. This is nearly the same as previously reported 3,55 .

We use tetragonal notation for all samples, with axes along the next-nearest-neighbor iron atoms. In the parent sample the lattice becomes orthorhombic below $T_{s}$, followed by ordering of the Fe magnetic moments below $T_{N}$. When required, we take the ferromagnetically (antiferromagnetically) ordered direction in the Fe plane to be parallel to $\langle 110\rangle(<1 \overline{1} 0\rangle)$ as shown in Fig. 1. The lattice constant along $<110>$ or the ferromagneti- 


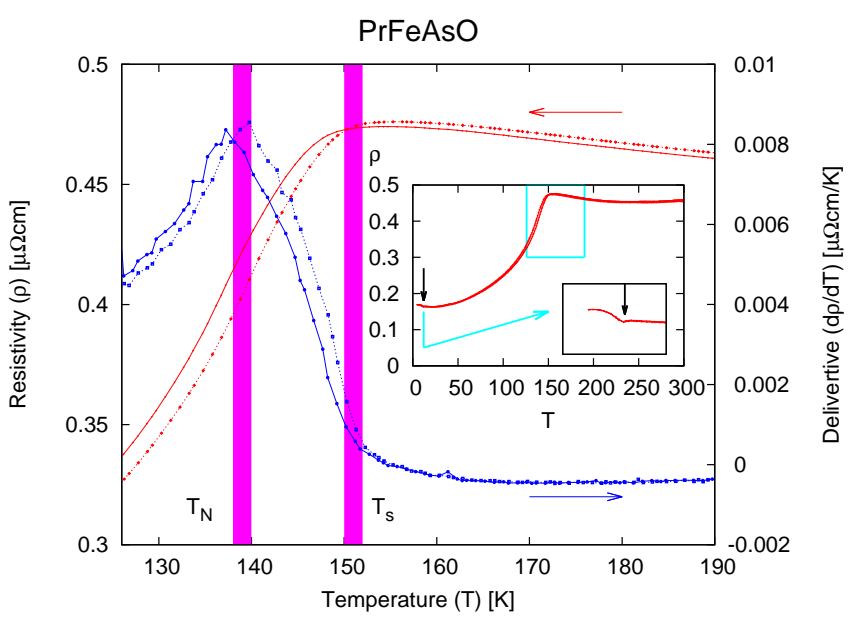

FIG. 2: (Color online) Electric resistivity $(\rho)$ and the derivative $(d \rho / d T)$ of a PrFeAsO sample near the structural and magnetic transition temperatures. Solid (dashed) lines correspond to the measurements with decreasing (increasing) temperature. Inset shows a larger temperature range for $\rho$. The deviation in $\rho$ from slow increase with decreasing temperature or an abrupt increase in $d \rho / d T$ corresponds to the structural transition temperature $T_{s}$ from tetragonal to orthorhombic symmetry, while the inflection point in $\rho$ or the maximum in $d \rho / d T$ corresponds to the magnetic transition temperature $T_{N}$. A small upturn below $12 \mathrm{~K}$ indicated by an arrow in the inset corresponds to the ordering of Pr magnetic moments.

cally ordered direction is shorter than that along $<1 \overline{1} 0>$ or the antiferromagnetically ordered direction, $a_{<110>}<$ $a_{<1 \overline{1} 0>}$.

\section{B. IXS measurements and data analysis}

IXS measurements were performed at BL35XU (Ref. 56) of SPring-8. The x-ray beam from the storage ring was first monochromatized to $\sim \mathrm{eV}$ by a double crystal, liquid nitrogen cooled, $\operatorname{Si}\left(\begin{array}{lll}1 & 1 & 1\end{array}\right)$ monochromator, and then down to $0.8 \mathrm{meV}$ using a $\mathrm{Si}(111111)$ backscattering monochromator operating at $21.75 \mathrm{keV}$. The incident beam was focused, by a bent cylindrical mirror, to a spot size of about 70 microns in diameter [full width at half maximum (FWHM)] at the sample position. The scattered radiation was analyzed using 12 spherical crystals in a 4 (horizontal) $\times 3$ (vertical) array on a horizontal 10 m $2 \theta$ arm. Each analyzer focused the radiation into a separate detector located near the sample.

The samples were mounted on thin glass rods, which were set on a small goniometer head in air for room temperature measurements, or on the cold finger of a ${ }^{4} \mathrm{He}$ closed-cycle refrigerator for low temperature investigations. The penetration depth of $\mathrm{PrFeAsO}_{1-y}$ for 21.75 $\mathrm{keV} \mathrm{x}$ rays is about $60 \mu \mathrm{m}$, which, with our thin samples, enabled us to measure in a transmission (Laue) geometry for many $Q$ points. The surface normal of all samples was along the $c$ axis, and they were aligned with the $<010>$ or $<1 \overline{1} 0>$ directions approximately vertical.

The use of the two-dimensional analyzer array allowed parallelization of measurements for either nearly pure longitudinal or transverse modes dispersing out from the $\Gamma$ point 57 . For example, with $<010\rangle$ vertical and the $<100>$ in the horizontal scattering plane, we can measure simultaneously four $Q$ points along approximately $<100>$ longitudinal direction or three $Q$ points along approximately $<010>$ transverse direction from a $\left(\begin{array}{lll}H & 0 & 0\end{array}\right)$ Bragg points using, respectively, a horizontal or vertical line of analyzers. Likewise, $<110>$ longitudinal and transverse modes can be investigated easily with the condition $\langle 1 \overline{1} 0\rangle$ vertical. The analyzers were not always perfectly centered along a symmetry direction, but the deviation was typically very small $[<0.02$ reciprocal lattice units (r.l.u.) in $H$ and $K$, and $<0.15$ r.l.u. in $L$ at the momentum transfers we measured]. A typical value for the momentum resolution (corresponding to the analyzer acceptance) is 0.06 r.l.u. in $H$ and $K$, and 0.12 r.l.u. in $L$.

To analyze the data, the obtained spectra were fit to the sum of a resolution-limited elastic peak and several Lorentzian phonon peaks:

$$
\begin{aligned}
& I(E)=A \operatorname{Res}(E) \\
& +\sum_{i} \frac{B_{i} b_{i}}{\pi}\left\{\frac{n\left(E+E_{i}\right)}{\left(E+E_{i}\right)^{2}+b_{i}^{2}}+\frac{n\left(E-E_{i}\right)+1}{\left(E-E_{i}\right)^{2}+b_{i}^{2}}\right\}(1)
\end{aligned}
$$

where $\operatorname{Res}(E)$ is the instrumental resolution function measured using elastic scattering from plexiglas, $A$ and $B_{i}$ are constants, $E_{i}$ and $b_{i}$ are the energy and the width of the $i$-th phonon peak, and $n(E)$ is the Bose thermal factor. Fitting to the above function was done using a nonlinear Levenberg-Marquardt least squares algorithm.

\section{First principles calculations}

Several first principles calculations of $\mathrm{PrFeAsO}_{1-y}$ and $\mathrm{LaFeAsO}$ were carried out using VASP $\underline{58}-60$ and a projector augmented-wave (PAW) method ${ }^{61,62}$, in the generalized gradient approximation (GGA), and local spin density approximation (LSDA). (Table III) Phonons were calculated via the PHONON package using a direct method $\underline{63}$. The supercell size used for each calculation is $2 a \times 2 a \times c$ (32 atoms) for "original", $2 a \times 2 a \times c(31$ atoms) for " $\mathrm{O}_{7 / 8}$ ", and $2 \sqrt{2} a \times 2 \sqrt{2} a \times 2 c$ (128 atoms) for both "magnetic" and "mag. tetra," where $a$ and $c$ are lattice constants of the tetragonal primitive unit cell (cf. Table【I). Total energies and inter-atomic forces were calculated for 16, 56, 30, and 30 symmetry-inequivalent displacements for original, $\mathrm{O}_{7 / 8}$, magnetic and mag. tetra calculations, respectively. The energy cut-off for plane waves was $550 \mathrm{eV}$, the spacing of $k$ points was less than $0.1 \AA^{-1}$ and the convergence condition was that the total energy difference be less than $1 \mu \mathrm{eV}$. All structures were relaxed. The structural parameters are summarized in Table II Stripe antiferromagnetic ordering, as observed 
TABLE II: Structural parameters from first principles calculations (upper portion) and the experiment (lower portion). "original" is the simplest calculation with nonmagnetic tetragonal structure, while " $\mathrm{O}_{7 / 8}$ " is a supercell calculation with a $12.5 \%$ ordered oxygen deficiency. "magnetic" and "mag. tetra" are the first principles calculations with antiferromagnetically ordered Fe moments (after Ref. 2), and the crystal symmetry is orthorhombic and tetragonal $\left(a_{\text {ortho }}=b_{\text {ortho }}\right.$; Ibam), respectively. "O $\mathrm{O}_{7 / 8}$ " gives different $z_{P r}$ 's and $z_{A s}$ 's and the averages are given. In the parent material below $T_{N}$ Fe spin moments are aligned ferromagnetically along the $b_{\text {ortho }}$ direction $(\|<110>$, the notation here as shown in Fig. 10).

\begin{tabular}{|c|c|c|c|c|c|c|c|c|c|}
\hline name & compound & symmetry & $a[\AA]$ & $a_{\text {ortho }} / b_{\text {ortho }}[\AA]$ & $c[\AA]$ & $z_{P r / L a}$ & $z_{A s}$ & $T[\mathrm{~K}]$ & \\
\hline original & $\mathrm{PrFeAsO}$ & $P 4 / n m m$ (tetra) & 4.0124 & $(5.6744)$ & 8.4863 & 0.14472 & 0.64052 & (0) & \\
\hline $\mathrm{O}_{7 / 8}$ & $\mathrm{PrFeAsO}_{7 / 8}$ & $P \overline{4} m 2$ (tetra) & 3.98635 & 5.6375 & 8.4336 & $<0.15466>$ & $<0.64367>$ & $(0)$ & \\
\hline magnetic & $\mathrm{LaFeAsO}$ & Ibam (ortho) & & $5.7320 / 5.6616$ & 8.6437 & 0.14359 & 0.64666 & $(0)$ & \\
\hline mag. tetra & $\mathrm{LaFeAsO}$ & $\operatorname{Ibam}$ (tetra) & $(4.0301)$ & 5.69937 & 8.7368 & 0.14269 & 0.64636 & $(0)$ & \\
\hline parent & $\mathrm{PrFeAsO}$ & & 3.976 & $(5.623)$ & 8.572 & & & R.T. & \\
\hline doped-1 & $\mathrm{PrFeAsO}_{0.9}$ & & $3.976(1)$ & $(5.623)$ & $8.5686(2)$ & & & R.T. & \\
\hline doped-2 & $\mathrm{PrFeAsO}_{0.7}$ & & 3.961 & $(5.601)$ & 8.539 & & & R.T. & \\
\hline \multirow[t]{3}{*}{ Powder } & $\mathrm{PrFeAsO}$ & $P 4 / n m m$ (tetra) & $3.97716(5)$ & $(5.6246)$ & $8.6057(2)$ & $0.1397(6)$ & $0.6559(4)$ & 175 & Ref. 4 \\
\hline & $\mathrm{PrFeAsO}$ & Cmma (ortho) & & $5.6374(1) / 5.6063(1)$ & $8.5966(2)$ & $0.1385(5)$ & $0.6565(3)$ & 5 & Ref. $\underline{\underline{4}}$ \\
\hline & $\mathrm{PrFeAsO}_{0.85}$ & P4/nmm (tetra) & $3.9686(1)$ & $(5.6124)$ & $8.5365(3)$ & $0.1450(7)$ & $0.6546(5)$ & 5 & Ref. $\underline{\underline{4}}$ \\
\hline
\end{tabular}

experimentally, is stabilized in the magnetic calculations, with structural parameters that are nearer to the experimentally determined values 64 .

Magnetic calculations were done using the La pseudopotential instead of $\mathrm{Pr}$ to avoid difficulties in treating the spins of the localized $\operatorname{Pr} f$ electrons in the core pseudopotential. However, a comparison of the results of nonmagnetic calculations using La and Pr showed the results to be nearly identical for the phonons, with the differences between the two calculations being much smaller than those between the calculation and the measured data. Considering a purely mass difference would suggest phonon frequency changes of less than 1 percent for $\mathrm{La} / \mathrm{Pr}$ modes.

\section{RESULTS}

\section{A. IXS measured data}

Figure 3 shows typical IXS spectra of parent $\mathrm{PrFeAsO}$ and doped $\mathrm{PrFeAsO}_{1-y}$ (doped-2; $T_{c}=45 \mathrm{~K}$ ) at room temperature at $Q=\left(\begin{array}{lll}3.03 & 0 & 0.06\end{array}\right)$ (near the Brillouin zone center, $\Gamma$ point) and (3.50 00.00$)$ (Brillouin zone boundary). An elastic peak and several phonons were observed, and fit using the function of Eq. (11). The sum fit curve as well as individual phonon lineshapes are shown in Fig. 3 .

Figure 4 shows the dispersion relations of $\mathrm{PrFeAsO}_{1-y}$ (parent: $y=0$, doped-1: $y \sim 0.1$ or doped-2: $y \sim 0.3$ ) at room temperature along some high-symmetry directions;

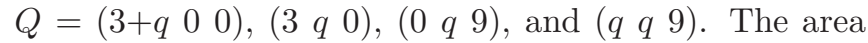
of the symbols is proportional to the integrated peak intensity, $B_{i}$, and the error bars give the intrinsic width, after subtracting the measured instrumental resolution width from $\left|b_{i} / 2\right|$. The uncertainty in mode energy is $<0.5 \mathrm{meV}$ except for some small and/or broad peaks.

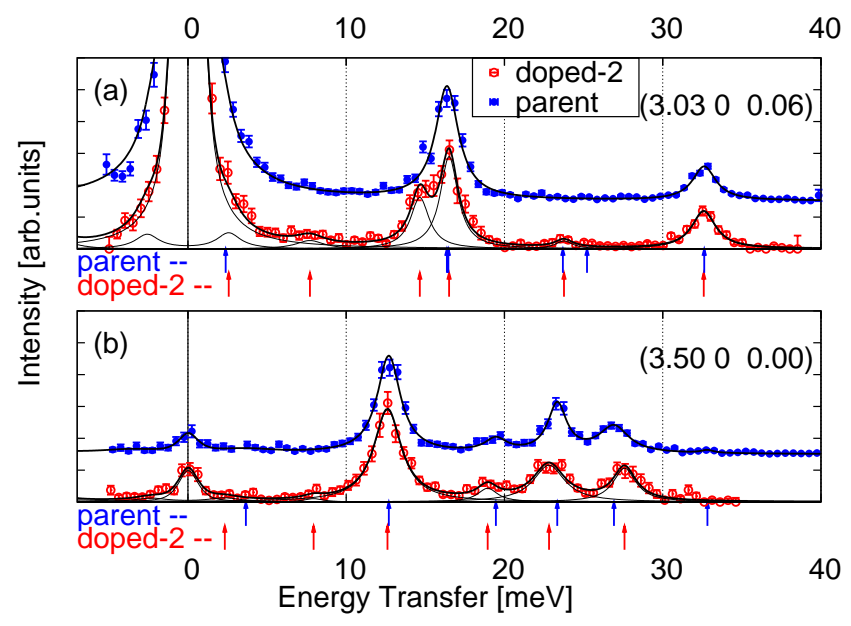

FIG. 3: (Color online) Typical IXS spectra of doped $\mathrm{PrFeAsO}_{1-y}$ (doped-2; open circle) and parent $\mathrm{PrFeAsO}$ (closed circle; shifted vertically for clarify) at room temperature (a) near $\Gamma$ point $(Q=(3.0300 .06))$ and (b) at Brillouin zone boundary $\left(Q=\left(\begin{array}{lll}3.50 & 0 & 0.00\end{array}\right)\right.$. The lines show the fit to the data using Eqn. (1). The individual peaks are also shown for the doped-2. The arrows below each plot show the peak positions based on the fits. Generally the elastic intensity is larger near to the $\Gamma$ point.

Though the data in doped $\mathrm{PrFeAsO}_{1-y}$ having slightly low $T_{c}$ (doped-1) are plotted only in Fig. प(b), the doped1 and doped-2 samples show exactly the same result at $Q=\left(\begin{array}{lll}3+q & 0 & 0\end{array}\right)$ (not shown).

There is a doping dependence, which is also branch dependent. For example, there are three strong branches observed at $Q=(3+q 00)$ in Fig. 4 (a). With doping, the branch dispersing from $\sim 32 \mathrm{meV}$ at $Q=\left(\begin{array}{lll}3 & 0 & 0\end{array}\right)$ to $\sim 27$ $\mathrm{meV}$ at $\left(\begin{array}{lll}3.5 & 0 & 0\end{array}\right)$ hardens, while the branch dispersing from $\sim 16 \mathrm{meV}$ at $\left(\begin{array}{lll}3 & 0 & 0\end{array}\right)$ to $\sim 23 \mathrm{meV}$ at $\left(\begin{array}{lll}3.5 & 0 & 0\end{array}\right)$ softens 


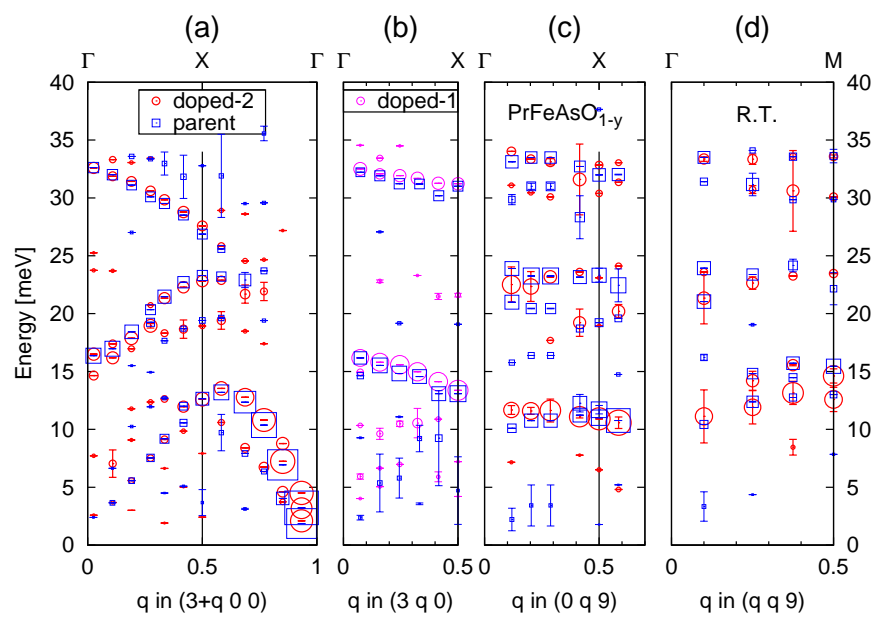

FIG. 4: (Color online) Dispersion relations along some highsymmetry directions of doped $\mathrm{PrFeAsO}_{1-y}$ (open circle) and parent $\mathrm{PrFeAsO}$ (closed circle) at room temperature. The $Q$ $=\left(\begin{array}{lll}3 & q & 0\end{array}\right)$ data of (b) are from the sample with $y \sim 0.1\left(T_{c}=\right.$ $36 \mathrm{~K}$; doped-1), and the other ones are from the sample with $y \sim 0.3\left(T_{c}=45 \mathrm{~K}\right.$; doped-2). The area of the symbols is proportional to the peak intensity $\left[B_{i}\right.$ in Eq. (1)] and the error bars are the intrinsic peak widths estimated by subtracting the experimental resolution from the fit result.

slightly. The hardening/softening may be larger at the zone boundary than at the zone center, which can be seen more clearly in Fig. 3. However, the differences are small $(\sim 0.5 \mathrm{meV})$, and there is no drastic change in the overall dispersion between the parent and doped sample data. The doping dependence in dispersion relations at low temperature below $T_{N}$ and $T_{c}$ show features similar to Fig. 目 (not shown).

Figure 5 shows the temperature dependence of the dispersion in the parent $\mathrm{PrFeAsO}$ at $Q=(3+q 00),(3 q 0)$, $(0 q 9)$, and $(q q 9)$. We can see small changes in some high energy branches. The mode energy increases with decreasing temperature, as may just be the result of thermal contraction. Measurements about $T_{s}$ (and $T_{N}$ ) near $Q=\left(\begin{array}{lll}3 & 0 & 0\end{array}\right)$ [Fig. [5(a)], did not show any strong changes on crossing the transition temperature, with mode frequencies being nearly unchanged and most mode intensities following the usual Bose factor. That is, the temperature dependence in doped superconducting $\mathrm{PrFeAsO}_{1-y}$ is similar to that in the parent sample, with a slight hardening as temperature is decreased, and no drastic change in the overall dispersion.

Measurements of the parent $\mathrm{PrFeAsO}$ were made along the $<110>$ direction at $10 \mathrm{~K}$, well below the measured $T_{N} \simeq 139 \mathrm{~K}$, to investigate possible effects of the orthorhombic structural distortion and appearance of magnetism. This direction potentially twins, with the $<110>$ and $<1 \overline{1} 0>$ directions becoming distinct. If the beam hits a twinned section of the sample, it would be reasonable (especially based on the magnetic calculations discussed below) to expect phonon splitting as the modes polarized

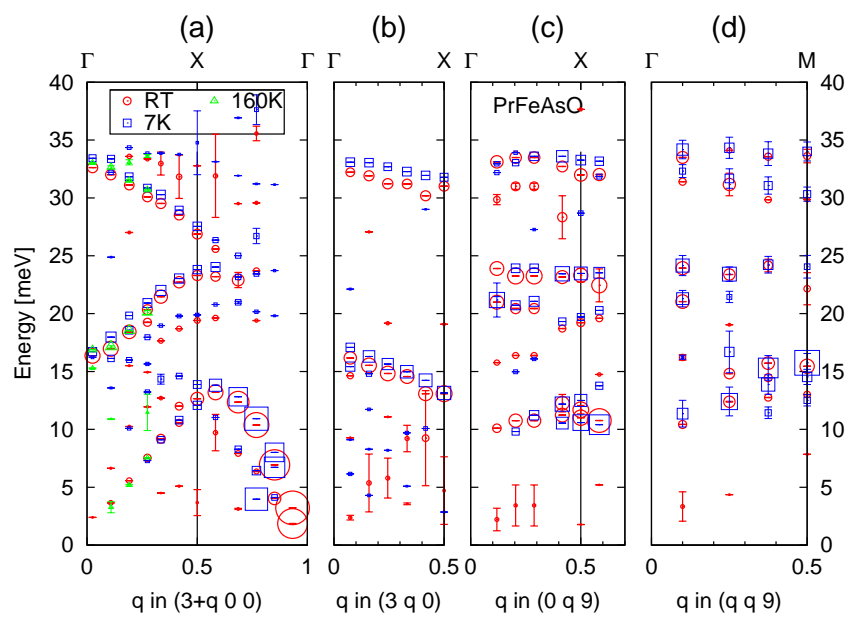

FIG. 5: (Color online) Dispersion relations along some symmetry directions for the parent $\mathrm{PrFeAsO}$. The figure style is the same as that of Fig. 4. The data at room temperature are identical to those in Fig. 4 .
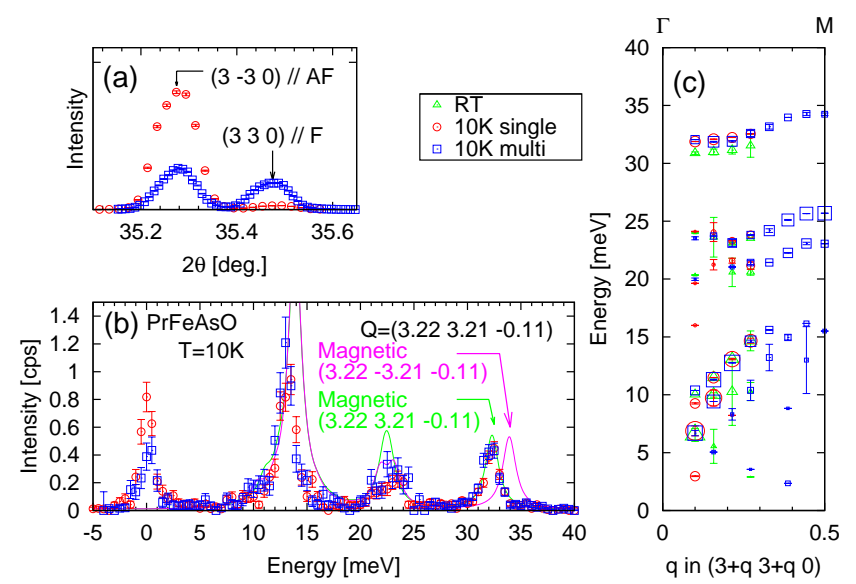

FIG. 6: (Color online) No magnetic splitting is observed in the phonon spectra for PrFeAsO at $10 \mathrm{~K}$. (a) $2 \theta$ scans near

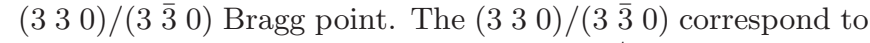
the scattering from antiferromagnetically/ferromagnetically ordered (orthorhombic $a / b$ ) direction, respectively. (b) Phonon spectra at $Q=(3.223 .21-0.11)$. The lines are the spin polarized $a b$ initio "magnetic" orthorhombic calculation, (see Sec. IIIB) convoluted with the instrumental resolution. (c) Dispersion relations.

in the $<110>$ ferromagnetic ordering direction (shorter lattice constant) could have different energies from those in the $<1 \overline{1} 0>$ antiferromagnetic ordering direction. With some effort, we were able to find part of the sample where twinning was clearly observed, based on the split of the (3 3 0) Bragg reflection [Fig. 6(a)], however, even in this twinned region, the phonon splitting near $\sim 34 \mathrm{meV}$ expected to be $\sim 3 \mathrm{meV}$ from magnetic calculation did not appear as shown in Fig. 6(b). The dispersion is plotted in Fig. 6(c) for the parent sample from two regions at 


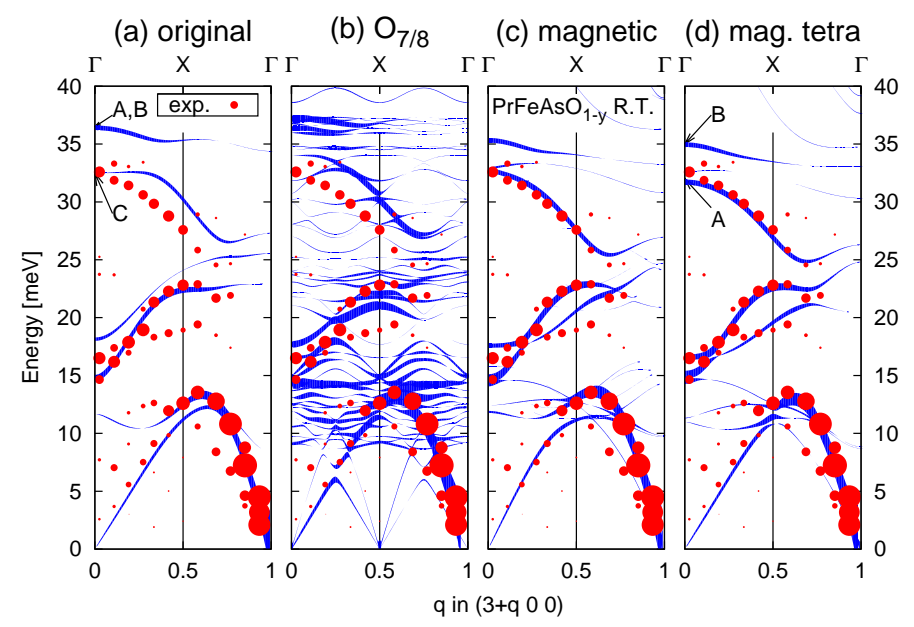

FIG. 7: (Color online) Comparison of the measured dispersion for $\mathrm{PrFeAsO}_{1-y}\left(T_{c}=45 \mathrm{~K}\right.$; doped-2) at room temperature at $Q=(3+q 00)$ and various first principles calculations. The same data are repeated in each panel for comparison with the different calculations: (a) "original" calculation using a nonmagnetic tetragonal structure, (b) the " $\mathrm{O}_{7 / 8}$ " calculation with an oxygen deficiency, (c) the "magnetic" first principles calculation, and (d) the tetragonal magnetic calculation. See also the text and table II for details. The area of the experimental data points (line height of the calculations) show the measured (calculated) mode intensity for IXS spectra. For clarity, a cutoff has been used in plotting acoustic mode intensity which becomes large near Bragg points. The low energy acoustic mode appearing at $(4-q 00)$ is probably a transverse mode. See text at the end of Sec. IIIB.

low temperature as well as those at room temperature. The spectral shape, and the dispersion is essentially identical. One notes that there is some hardening with decreasing temperature observed in the high energy branch [Fig. 6(c)] and almost no doping dependence in this direction (not shown). This small doping/temperature effects are similar to other phonon modes discussed above (Figs. 4 and 5 ).

\section{B. Comparison with ab initio calculations}

We now compare the dispersion against the unmodified ab initio calculations. Since the effect of carrier doping, or sample temperature, on the phonon spectra is relatively small (see Figs. 4 and 5 ) we plot only one data set, that from the superconducting $\mathrm{PrFeAsO}_{1-y}$ (doped-2). Figure 7 shows the comparison between the experimental data and the calculations at $Q=(3+q 00)$. Again, the area of experimental data points shows the mode intensity after removal of the Bose factor. For the calculations, the line height shows the expected intensity. The scale factor (symbol area to line height) is fixed for all data sets. The experimental data in Fig. 7 are the same as shown previously [doped-2 in Fig. [4(a)], but the error bars indicating the intrinsic peak width are omitted.
The agreement between the data and calculation is fairly good for low energy branches. However, the branch dispersing from $\sim 33 \mathrm{meV}$ at the $\Gamma$ point shows notable differences between the different calculations. The measured energy is generally significantly lower than that of the first principles calculation with a nonmagnetic ground state [Fig. 7(a) "original"], consistent with our earlier work ${ }^{37}$. As the superconducting samples are oxygen deficient, we also made calculations with a deficiency (12.5\%) using a large unit cell size $2 \times 2 \times 1$ with one oxygen removed [Fig. 7 (b) "O $\mathrm{O}_{7 / 8}$ "]. The agreement with the experimental data is similar to the "original" calculation, but many added branches appear, as might be expected from adding an ordered deficiency. Such additional modes are not observed in our measurement, and the agreement with the high energy branch in the "original" calculation is not improved. This calculation then mostly provides confirmation that the oxygen deficiency probably does not have a surprising impact on the detailed phonon dispersion. This is consistent with the recent first principles calculation on the doping dependence of phonon DOS in $\mathrm{LaFeAsO}_{1-x} \mathrm{~F}_{x}$ using the virtual crystal approximation ${ }^{34}$.

We also carried out calculations with antiferromagnetically ordered Fe moments in an orthorhombic structure,

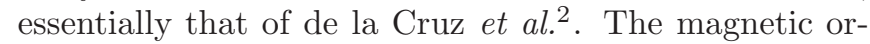
der of Pr atoms was not considered as they were substituted with La in the calculation. The result is plotted in Fig. Z(c) (magnetic). The agreement with the experimental data is better than the "original" model. We also performed a calculation of a tetragonal magnetically ordered material to distinguish between the effects of the magnetic moment and crystal symmetry. The result is plotted in Fig. 7(d) (mag. tetra), and it is very similar to the "magnetic" calculation. This clearly shows the phonon softening of the branch at $27-33 \mathrm{meV}$ in the first principles calculation comes from allowing the magnetic order of the iron atoms: the orthorhombic/tetragonal crystal symmetry change has a relatively small effect on the calculated phonon dispersion.

The addition of the magnetism, at first glance, improves the agreement between the data and the calculations. It is worth emphasizing that this is true for both the parent material (data not shown) which explicitly shows magnetic order, and also the superconducting materials [data as shown in Fig. [7(c)] which do not show evidence of static magnetic order. The latter is somewhat surprising, and we will discuss it later again. However, while the magnetic calculations do better, they also predict splitting of modes that is much larger than that observed in our data. This is evident in Figs. 7(c) and (d), where calculations give a high energy branch $(\sim 34 \mathrm{meV})$ that is not observed here. The high energy branch originates from the magnetic calculation lifting a degeneracy between the ferromagnetically and antiferromagnetically polarized modes: the measured phonon energies are consistent with the energies calculated for modes with $\mathrm{Fe}$ motions in the ferromagnetic ordering direction [motion 


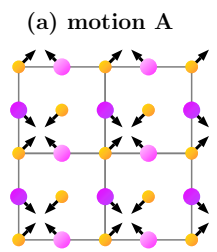

(d) motion D

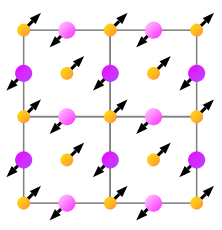

(f) motion $\mathrm{F}$

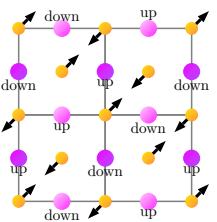

(h) motion $\mathrm{H}$

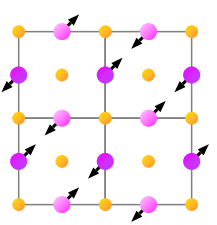

(b) motion B

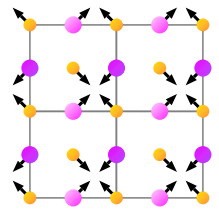

(e) motion $\mathrm{E}$

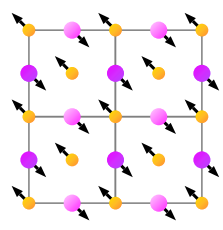

(g) motion G

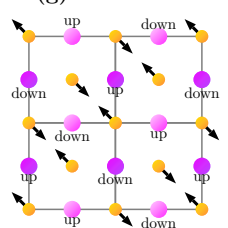

(i) motion I

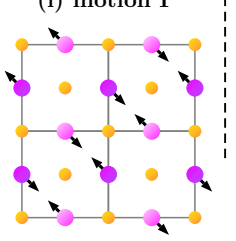

(c) motion $\mathrm{C}$
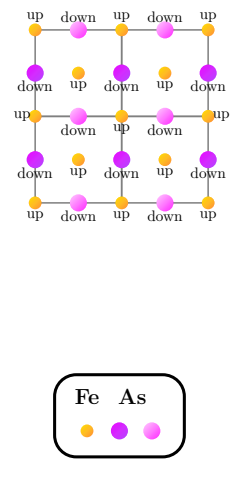

AF spin arrangement
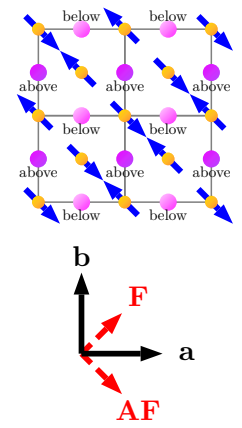

FIG. 8: (Color online) The bottom right is a schematic of the Fe-As layer: As atoms are located above or below the Fe atomic plane as specified. The arrows on Fe atoms specify the magnetic spin directions for "magnetic" and "mag. tetra" calculations, where the ferromagnetic (antiferromagnetic) propagation vector corresponds to be $<110>(<1 \overline{10}>)$ as shown in the red dashed arrow $\mathrm{F}(\mathrm{AF})$. In (a)-(i) arrows specify atomic displacement patterns in an Fe-As layer at several points in Figs. 7]and 9] Atomic movements along out-of-plane direction are specified by "up" or "down" near the atoms.

A in Fig. $8(a)$ but not with the calculated energies of modes with $\mathrm{Fe}$ motions in the antiferromagnetic direction, of which should also appear in the $(3+q 00)$ direction [motion B in Fig. \&(b)].

The failure of the magnetic calculations is further confirmed by investigating dispersion along the $\langle 110\rangle$ direction. A splitting similar to that mentioned above is calculated to appear between ferromagnetically polarized modes (observed in a longitudinal $<110>$ geometry) and antiferromagnetically polarized modes (observed in the $<1 \overline{1} 0>$ ) in a twinned portion of the parent below $T_{N}$. As discussed previously, Fig. [6 shows the measurement of longitudinal modes in the $<110>$ direction, from both a single domain and a twinned section of the crystal. The phonon spectra are essentially identical, showing no evidence of the calculated splitting.

The lack of splitting is also confirmed in Fig. 9 along $(3+q 3+q 0)$ corresponding to Fig. 6(c). In the figure, the experimental data are compared with (a) "orig-

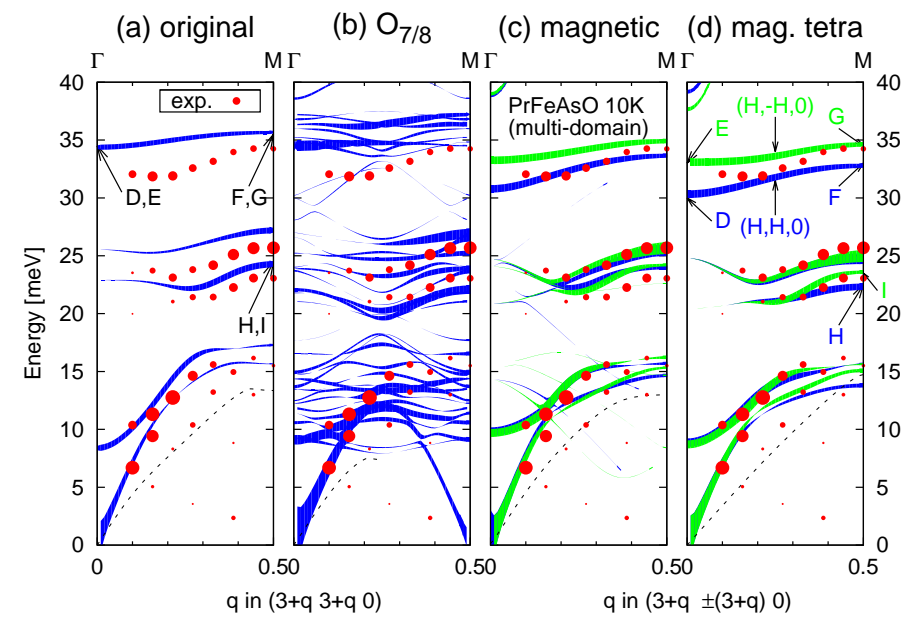

FIG. 9: (Color online) Dispersion along $Q=(3+q 3+q 0)$ for the parent sample PrFeAsO. In this direction of (c) "magnetic" and (d) "mag. tetra" calculations there are two domains originated from crystal structure and/or magnetic order, and the phonon dispersions from each domains are plotted. See the caption of Fig. 7 for details. The weak, low energy, acoustic mode that can be seen in the data is a transverse acoustic mode (dashed line) that appears in the measured spectra, probably due to the finite momentum acceptance of the analyzers. See the text at the end of Sec. IIIB for discussion.

inal", (b) "O $\mathrm{O}_{7 / 8}$ ", (c) "magnetic", and (d) "mag. tetra" calculations. The $\langle 110\rangle$ and $\langle 1 \overline{1} 0\rangle$ directions are not equivalent, as is shown by choosing different colors (gray scales). The general features are consistent with those of $Q=\left(\begin{array}{lll}3+q & 0 & 0\end{array}\right)$ in Fig. 7 in that the branches at low energy are explained fairly well by any calculation, while the branches at the energy of $30-35 \mathrm{meV}$ are not. A similar feature is also obtained in the data along other symmetry directions of $Q=(3 q 0),(0 q 9)$ and $(q q 9)$, which are corresponding to (b)-(d) in Figs. 4 and 5 ,

Figure 8 investigates the calculated polarizations related to the splitting of ferromagnetically and antiferromagnetically polarized modes in more detail, showing the atomic motions at $\Gamma$ and $\mathrm{M}$ points. For these modes only $\mathrm{Fe}$ and $\mathrm{As}$ atoms move, while rare-earth $(\mathrm{La} / \mathrm{Pr})$ and $\mathrm{O}$ atoms do not. The motions $\mathrm{A}$ and $\mathrm{B}$ are degenerate in the "original" calculation, and split in "magnetic" and "mag. tetra" calculations. The pairs D and E, F and $\mathrm{G}$, and $\mathrm{H}$ and $\mathrm{I}$ are the same as the pair $\mathrm{A}$ and $\mathrm{B}$. In each pair the phonon energy of motions $\mathrm{A}, \mathrm{D}, \mathrm{F}$, and $\mathrm{H}$ is lower than that of motions B, E, G, and I. In all cases, the motion containing the vibration of Fe atoms along the $<110>$ direction (ferromagnetic mode) has lower energy than that along the $<1 \overline{1} 0>$ direction (antiferromagnetic mode) excepting those modes without in-plane Fe motion (i.e., $\mathrm{H}$ and I where Fe atoms do not vibrate).

Finally we note the appearance of a transverse mode in the longitudinal spectra, as can be seen in Fig. 9. The predominantly transverse character of the mode is confirmed by its higher intensity in the "off symmetry" 
TABLE III: Modified model calculations. See text for discussion and Fig. 10 .

\begin{tabular}{ccl}
\hline \hline name & based ab initio calc. & modification \\
\hline soft Fe-As & "original" & weaken Fe-As \\
in-plane soft & "original" & weaken in-plane Fe-As \\
clipped & "magnetic" & cut Fe-Fe along AF \\
\hline \hline
\end{tabular}

analyzers (data not shown) which have a larger transverse contribution. Its appearance, in principle, then can be explained by the finite momentum acceptance of the analyzers [for example $\Delta Q \simeq 0.029$ r.l.u. near the $Q=$ $\left(\begin{array}{lll}3 & 0 & 0\end{array}\right)$. However, the investigation of the calculations in both this section, and the next, shows that there is also the potential that the mode itself may be of mixed polarization. Furthermore, the amount of mixing might be some way of selecting between different calculations, though such a detailed analysis, relying heavily on both the intensity and momentum resolution is beyond the scope of the present work.

\section{COMPARISON WITH MODIFIED MODELS}

The "magnetic" calculations provide the best over-all agreement with our data out of those presented above. However, there remain significant discrepancies with the calculations predicting mode splitting that is not observed experimentally as discussed above. To better understand these continued discrepancies, we consider several direct modifications to the real-space force constant matrices resulting from (the interpolation of) the $a b \mathrm{ini}$ tio calculations. Since the discrepancies between the calculations and the data remain generally larger than those between different dopings or temperatures, we focus on modifying the models to get globally similar characteristics to the data. The models are summarized in Table III and Fig. 10, For all of them we preserved the crystal symmetry, and recalculated the self forces to keep the translational invariance. Optimizations were done by hand.

The simplest model we consider is the "soft Fe-As" model of Ref. 37 where the nearest neighbor Fe-As force constant was scaled (reduced) by $30 \%$ [Fig. 10(a)-1] from that of the nonmagnetic 'original' calculation. This was shown to reproduce the softening of the peak in the DOS, but, not completely the dispersion in our previous work $^{37}$. The comparison with the in-plane longitudinal mode along $\langle 100\rangle$ is shown in Fig. 111(a). While the high energy branch is softened, the other branches are almost unchanged. The agreement with the experiment improves compared to the "original" calculation, but the branch shape is clearly different, with an anti-crossing in the calculation that is not present in the measurements.

It is possible to modify the nonmagnetic tetragonal ("original") calculation to agree better with the observed (a)

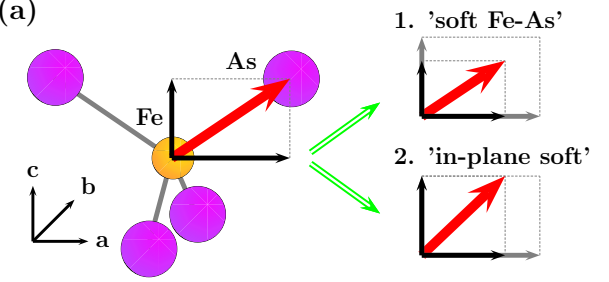

(b) force constant (c) magnetic correlation 'clipped' model
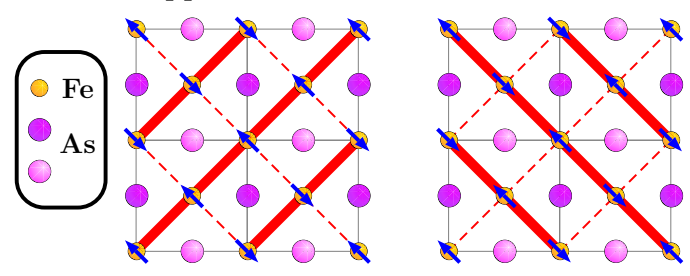

FIG. 10: (Color online) Schematic of model modifications. (a) The "soft Fe-As" model softens both the in-plane and the outof-plane components, while the "in-plane soft" reduces only the in-plane correlation. (b) Strength of the force constants between Fe atoms on an Fe-As layer in the "clipped" model. A strong correlation exists along the ferromagnetic direction as shown by the thick lines. (c) Effective magnetic exchange coupling constants between $\mathrm{Fe}$ atoms in an Fe-As layer suggested by spin wave measurement in $\mathrm{SrFe}_{2} \mathrm{As}_{2}$ (Ref. 65) and $\mathrm{CaFe}_{2} \mathrm{As}_{2}(\operatorname{Ref}$. 66). Strong interaction (large $J$ ) is along the direction of antiferromagnetic order.

dispersion by softening only the in-plane components of the nearest neighbor Fe-As force constant matrix. The "original" model failed to fit the data, because (1) the in-plane polarized mode at $\Gamma$ point (A and B in Fig. 7 with atomic motions $\mathrm{A}$ and $\mathrm{B}$ in Fig. 8) has an energy that is too high compared to the data, and (2) as one increases $q$ along the $<100\rangle$ direction, the model predicts an anti-crossing with a $c$-axis polarized mode $(\mathrm{C}$ in Fig. 7 and the atomic motion $\mathrm{C}$ in Fig. 8) that is not observed. However, selectively reducing the in-plane components of the Fe-As force constant matrix by $20 \%$ reduces the energy of the in-plane modes to be equal or just below that of the $c$-axis polarized mode, and avoids the anti-crossing, in good agreement with the measured data. This "in-plane soft" model [Fig. 10(a)-2] is compared with the experimental data in Fig. 111(b), and, for being a relatively simple modification, agrees well with the measured dispersion. The improvement is also clearly seen along $(3+q 3+q 0)$ in Figs. 12(a) and (b). In particular this direction shows the necessity of softening only the in-plane components instead of the full force constant matrices as we did in Ref. 37 .

Our third modified model begins with the force constant matrices from the "magnetic" first principles calculation. To reduce the splitting between ferromagnetic modes (whose calculated frequencies agree with the data) and the antiferromagnetic ones (which are calculated to have a higher energy than observed), we reduce the $<1 \overline{1} 0>$ components of force constant matrices between 
(a) soft Fe-As

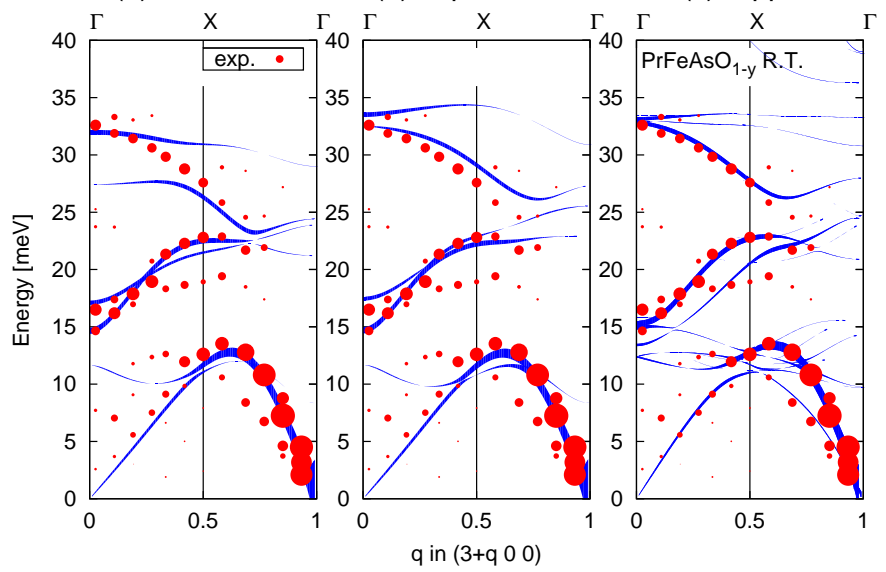

FIG. 11: (Color online) (a) Comparison of the experimental data of $\mathrm{PrFeAsO}_{1-y}\left(T_{c}=45 \mathrm{~K}\right.$; doped-2) at $Q=(3+q 00)$ at room temperature with the "soft Fe-As" model, (b) the comparison with "in-plane soft" model, and (c) the comparison with "clipped" model. The area of the experimental data as well as the line width of the calculations show the peak intensity on IXS spectra. See text for details. (a) soft Fe-As

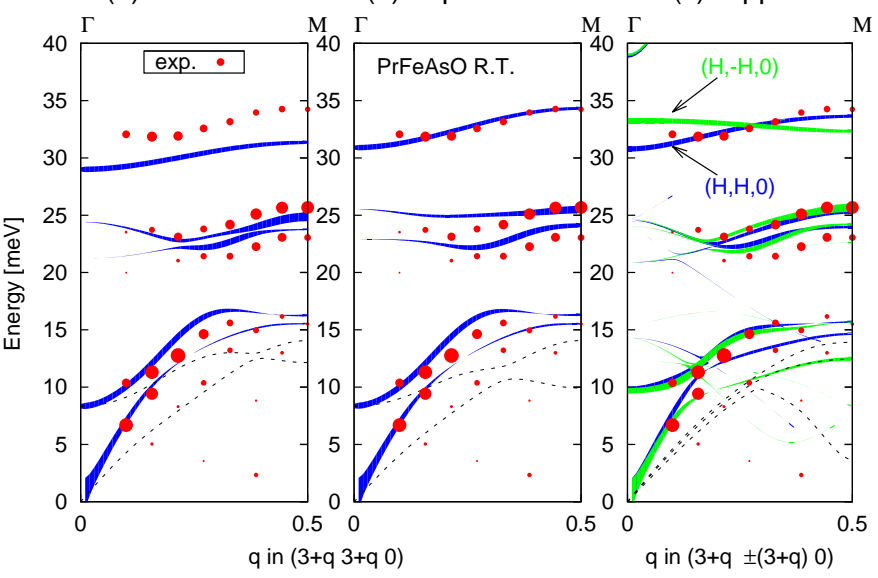

FIG. 12: (Color online) As Fig. 11 but the data are from parent $\mathrm{PrFeAsO}$ and along $Q=(3+q 3+q 0)$. In (c) "clipped" model the $\langle 110\rangle$ and $\langle 1 \overline{1} 0\rangle$ directions are inequivalent and are shown in different colors (gray scales).

nearest neighbor Fe atoms. Since the force constant between nearest neighbor Fe atoms is much smaller (less than $15 \%$ ) than that between nearest neighbor Fe and As, we "clip" these bonds by setting the force constant matrix to zero as shown in Fig. 10(b). This "clipped" model is compared with the experimental data in Fig.111(c), and the agreement is also relatively good.

The "in-plane soft" and "clipped" models have reasonable, though not perfect, agreement with the experimental data in other symmetry directions. For the dispersion along the $(3+q 00)$ (Fig. [11) and $(0 q 9)$ (not shown) the "clipped" model is perhaps slightly better, while for the

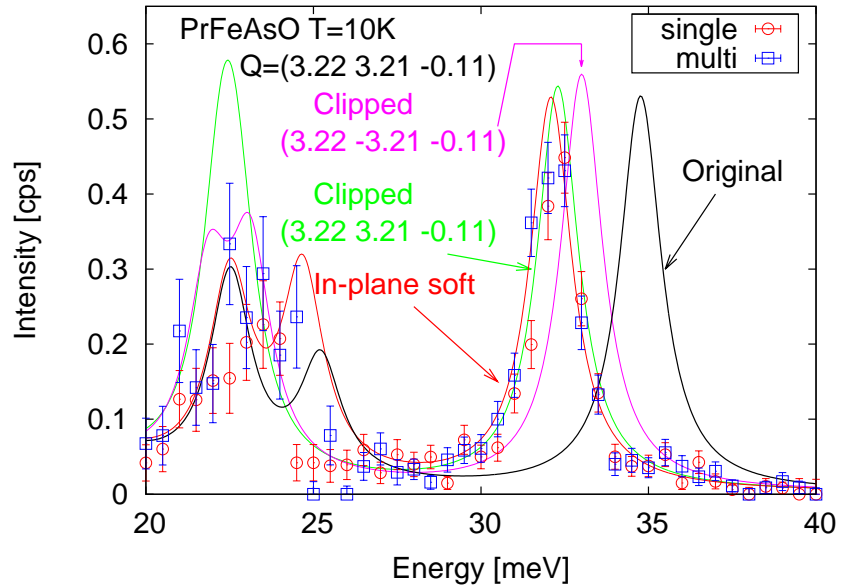

FIG. 13: (Color online) IXS spectra at $Q=(3.223 .22-0.11)$ in parent $\mathrm{PrFeAsO}$ sample at $10 \mathrm{~K}$ around the energy transfer of $30 \mathrm{meV}$; magnification of Fig. 6(b). The symbols are also the same as those in Fig. 6. While the red open circles show the measurement at a mostly single domain region, blue open squares show the measurement at a multi domain region. Two modified models as well as the "original" first principles calculation are also plotted with lines.

(3 $q$ 0) direction (not shown) the "in-plane soft" model is better. However, if we consider the splitting in the $(3+q 3+q 0)$ direction of Fig. 12, we find that the "clipped" model continues to predict some splitting that is not observed experimentally. This is also seen in the comparison of the IXS spectra of the parent sample below $T_{N}$ at single and multi domain regions with the "original" $a b$ initio calculation and two modified models in Fig.13.

\section{DISCUSSION}

The discussion above, after extensive investigation of several samples of $\mathrm{PrFeAsO}_{1-y}(y \sim 0,0.1$, and 0.3$)$ and comparison with $a b$ initio calculations and the modified models, allows the following conclusions:

1. There are small changes in the observed phonon intensities and dispersion when temperature or doping is modified.

2. The observed changes are generally much smaller than the rather large differences between the observed data and the $a b$ initio calculations.

3. Magnetic (spin-polarized LSDA) calculations tend to give better agreement with the measured data, because ferromagnetically polarized modes are softened. However, antiferromagnetically polarized modes are calculated to have energies that remain high and do not agree with measurement. 
4. Better agreement with the data can be obtained by modifying the calculation either by clipping the bond between antiferromagnetically polarized iron atoms in spin-polarized calculations, or by softening only the in-plane components of the force constant matrix of the Fe-As bond in nonmagnetic calculation.

We now discuss these results in the context of other work. At the start we note that variations of 13 have already been reported for other iron-arsenide compounds. Our work then demonstrates the general applicability of these conclusions across many families of iron-arsenide compounds. We also have put some results on rather firmer footing (e.g. the lack of splitting of modes in explicitly twinned material). Moreover, after a precise comparison with "magnetic" first principles calculations, which had been previously considered to show good agreement with experimental data by others, we still find significant differences. Consideration of these differences leads to two modified models summarized in the above point 4

The presence of only small changes in phonon spectra resulting from either temperature or doping, is in agreement with work by many other authors. Phonon DOS measurements by either INS or IXS have been carried out in various iron-arsenide compounds; "1111" materials $\left[\mathrm{LaFeAs}(\mathrm{O}, \mathrm{F})\right.$ (Refs. 35 37), $\mathrm{PrFeAsO}_{1-y}$ (Ref. 37), $\mathrm{NdFeAs}(\mathrm{O}, \mathrm{F})$ (Ref. 38), $\mathrm{Ca}(\mathrm{Fe}, \mathrm{Co}) \mathrm{AsF}$ (Ref. 46), and SrFeAsF (Ref. 51)], "122" materials [(Ba,K)Fe $\mathrm{Fe}_{2}$ (Ref. 39), (Ca,Na)Fe $\mathrm{As}_{2}$ (Ref. 40), $\mathrm{Ca}(\mathrm{Fe}, \mathrm{Co})_{2} \mathrm{As}_{2}$ (Ref. 41), and $\mathrm{SrFe}_{2} \mathrm{As}_{2}$ (Ref. 51)], and "11" system $\left[\mathrm{FeSe}_{1-x}\right.$ (Ref. 43)]. Fe partial phonon DOS measurements by nuclear resonant inelastic scattering of synchrotron radiation is adapted to $\operatorname{LaFeAs}(\mathrm{O}, \mathrm{F})$ (Ref. 67), (Ba,K)Fe ${ }_{2} \mathrm{As}_{2}$ (Ref. 68), $\mathrm{Ba}(\mathrm{Fe}, \mathrm{Co}) \mathrm{As}_{2}$ (Ref. 69), and $\mathrm{Fe}_{1+\delta} \mathrm{Se}$ (Ref. 70). Using single crystals precise phonon dispersions have been investigated by either IXS or INS in $\mathrm{PrFeAsO}_{1-y}$ (Ref. 37), (Ba,K)Fe $\mathrm{Fe}_{2} \mathrm{As}_{2}$ (Ref. 48), and $\mathrm{SmFeAs}(\mathrm{O}, \mathrm{F})$ (Ref. 49). All of them found only rather small changes, if any, with doping and temperature. The exception is the change in $(\mathrm{Ba}, \mathrm{K}) \mathrm{Fe}_{2} \mathrm{As}_{2}$ (Ref. 44 ), but it is observed in only one particular phonon mode. Though $(\mathrm{Ca}, \mathrm{Na}) \mathrm{Fe}_{2} \mathrm{As}_{2}$ also shows some changes in phonon structure with pressure ${ }^{42}$, this system is a relatively soft material with a structural transition to "collapsed tetragonal" phase. Raman ${ }^{71-84}$ and infrared (IR) spectroscopy ${ }^{85-88}$, or femtosecond-resolved pump-probe reflectivity ${ }^{89}-91$, are also used to investigate phonons in these materials, and no significant change is observed. Our result of a generally weak dependence of the phonon spectra on doping and temperature is then reasonably consistent with previous work. Higher resolution work does show some changes typically at the level of $0.5 \mathrm{meV}$.

Compared to the small temperature/doping dependences, the discrepancies between the measured data and the $a b$ initio calculations are relatively large (i.e., a softening of observed Fe modes). Moreover, the discrepancies become smaller if magnetism is included in the calculations. In general, this is also in good agree- ment with previous results. Many phonon DOS as well as phonon dispersion measurements show the softening of the experimental data compared with nonmagnetic $a b$ initio calculation by several meV on selected Fe and As phonon modes $36-38,45-51,92$. Among them some report the improvement possible using magnetic calculations; phonon DOS of $\mathrm{BaFe}_{2} \mathrm{As}_{2}$ (Refs. 92, 93), $R e \mathrm{Fe}_{2} \mathrm{As}_{2}$ and ReFeAsF (Ref. 51), phonon dispersion of $\mathrm{CaFe}_{2} \mathrm{As}_{2}$ (Ref. 47), $\operatorname{SmFeAs}(\mathrm{O}, \mathrm{F})$ (Ref. 49), (Ba,K)Fe ${ }_{2} \mathrm{As}_{2}$ and $\mathrm{Ba}(\mathrm{Fe}, \mathrm{Co})_{2} \mathrm{As}_{2}$ (Refs. 45, 48). Here we note that the most pronounced softening is observed for high energy plane-polarized modes $(\Delta E \sim 30-35 \mathrm{meV})$ in our present work, while most of the previous results were focused to $\Delta E \sim 20$ meV especially in the phonon dispersions.

Given the generally good agreement of our calculations of the ferromagnetically polarized modes with the measurements on the magnetically ordered parent below $T_{N}$, the remaining discrepancy between the calculated high energy for the antiferromagnetically polarized modes and the measurements which show a lower energy (essentially the same as the ferromagnetically polarized modes) is surprising. This is a strong result in that essentially all of our measurements only show the lower energy modes, and is highlighted by the complete lack of splitting (to the $0.5 \mathrm{meV}$ level) for the occasion when we specifically examined a twinned portion of the sample. A similar lack of splitting was also observed by Reznik et al. $\stackrel{48}{\text { for }}$ $\mathrm{BaFe}_{2} \mathrm{As}_{2}$, though without explicit confirmation of twinning.

We tried to understand the disagreement of data and calculations by ad hoc modifications to our calculation results, and arrived at two models, which have a completely different approach, but both of which show relatively good agreement with the observed data.

First we consider the "clipped" model. In this model we use the "magnetic" calculation, and include additional in-plane anisotropy to cancel the anisotropy originating from the magnetic order. The observed in-plane phonon dispersion of even antiferromagnetically ordered phase is surprisingly isotropic in the Fe-As plane. Therefore, additional anisotropy in the force constant matrices is indispensable, if we based it on "magnetic" calculation which it is now widely believed to be the reasonable calculation of the iron-arsenide system. The anisotropy in the FeAs plane, included in "clipped" model, is also suggested by other experiments. For example, from magnetic excitation measurements by INS large anisotropic exchange coupling is suggested in $\mathrm{CaFe}_{2} \mathrm{As}_{2}\left[S J_{a}=49.9 \pm 9.9\right.$ and $S J_{b}=-5.7 \pm 4.5$ (Ref. 66) or $24<J_{a}<37$ and $7<J_{b}<20$ (Ref. 94)] or in $\mathrm{FeSe}_{0.5} \mathrm{Te}_{0.5}$ (Ref. 95). For these the strong correlation is along the ferromagnetic direction in the "clipped" model [Fig. 10(b)], while the spin-spin coupling is stronger in the antiferromagnetic direction [Fig. 10(c)]. Moreover, recently orbital ordering in iron-pnictides has been discussed. Using the polarized laser angle-resolved photoemission spectroscopy (ARPES) two-fold symmetry is observed in electronic structure of the Fe $3 d_{x z}$ orbital in $\mathrm{BaFe}_{2} \mathrm{As}_{2}$ be- 
low $T_{N}$ (Ref. 96). Such orbital ordering also causes inplane anisotropy and even might explain 97 the abovementioned in-plane magnetic anisotropy of the spin waves. These anisotropic effects seem to suggest some support for the "clipped" model. However, the residual anisotropy of mode energies in the "clipped" model is larger than observed, and attempts to reduce it to the level measured (e.g., by including an attractive term) are not successful. Given the evidences for anisotropy (e.g., in spin wave 66.94 , transport measurements ${ }^{98}$, small $(\sim 0.5$ meV) splitting of phonon modes in Raman scattering ${ }^{76}$ ) it seems probable that the reality is a nearly isotropic model, with some very small anisotropy. However, the reason the direct $a b$ initio calculations so strongly overestimate the anisotropy is not clear.

The "in-plane soft" model is an alternative based on the "original" microscopically in-plane isotropic calculation. We slightly soften only the in-plane components of force constants while preserving the out-of-plane component. This is an improved version of our previous "soft Fe-As" model ${ }^{37}$, based on the nonmagnetic calculation. However, it shows as good as or even better agreement with the measured phonon structure compared to the "clipped" model. Therefore, it seems worth considering nonmagnetic calculations again, because experimentally the magnetic order does not strongly affect the phonon structure, and the dispersion is essentially isotropic in many iron-arsenides.

Finally we consider the effect of magnetic fluctuations, which is widely discussed and has the possibility to reduce the in-plane anisotropy. One model by Mazin and Johannes ${ }^{99}$, suggested there are always antiferromagnetic domains, but the boundaries fluctuate. Thus depending on the time scale of an experimental probe relative to these fluctuations, different determinations may be made about the presence of magnetism. They suggested that many of the physical properties of iron-arsenide compounds, both experimental and theoretical, may be explained by this. With this model the small observed Fe magnetic moment is explained as by averaging over zero-point and fast magnetic domain motion. Thus, Mössbauer experiments $\frac{67,100}{}$ and $\mu \mathrm{SR}^{101-104}$, that probe relatively slow (>10 ns) timescales would see primarily nonmagnetic response, as they do, while probes of faster time scales might see magnetic response, such as the splitting observed in photo-emission ${ }^{105}$. INS investigations, in fact, show magnetic fluctuations at finite energy $(\sim 0.2$ ps time scales) even in superconducting samples and/or at high temperature above transition 106-108.

However, it seems unlikely to us that the presence of fluctuations can fully explain the difference between our calculations and our data. Phonon time scales are given by the oscillation period ( $\sim 0.1 \mathrm{ps}$ for a $30 \mathrm{meV}$ mode), and the phonon lifetime ( $\sim 0.7 \mathrm{ps}$ for a $1 \mathrm{meV}$ mode) linewidth. Fluctuations on that are slow on the ps time scale should lead to splitting of modes, while those with timescales $<0.1$ ps should lead to unsplit phonons that see the average structure. The magnetic calculations give mode splittings of several meV while for the particular case of Fig. 7. for example, our data would support splittings no larger than a few tenths of a meV. Also, the observed mode energy is in good agreement with the one of the two calculated modes, not their average. Thus, we speculate there is some additional neglected factor, not only magnetic fluctuations, that leads to the overestimation of the calculated phonon anisotropy in these systems.

\section{CONCLUSION}

Extensive investigation of phonon dispersion in several samples of $\mathrm{PrFeAsO}_{1-y}$ allow careful comparison of results between different samples, and with calculations. Small changes were visible as the material was doped into the superconducting state (from $y=0$ to $y \sim 0.3$ ), and when the parent materials was cooled below the magnetic and structural phase transition. Smaller changes were visible when superconducting samples were cooled across $T_{c}$. Interpretation of the observed small changes with doping or temperature in this system is complicated by the generally larger disagreement with calculations. Magnetic calculations compare more favorably to the data, especially the ferromagnetically polarized phonon modes, but show splitting between ferromagnetically and antiferromagnetically polarized modes (several $\mathrm{meV}$ ) that is not at all supported by the experimental data $(<0.5 \mathrm{meV})$. Interestingly, the in-plane isotropy of phonon properties can be constructed from both microscopically anisotropic (magnetic) and isotropic (nonmagnetic) calculations. It is clear that while the $a b$ initio calculations provide a reasonable rough estimate of phonon dispersion, they lack some fundamental ingredient. Given the similarity of the measured response for both the magnetically ordered parent material below $T_{N}$ and the other systems, however, is seems unlikely that the missing ingredient might be only magnetic fluctuations.

\section{Acknowledgments}

Work at SPring-8 was carried out under proposal No. 2008A2050, No. 2008B1403, No. 2009A1436, No. 2009B1609, No. 2009B2136, and No. 2010A1296. This work was performed under the NIMS-RIKEN-JAEA Cooperative Research Program on Quantum Beam Science and Technology. 
1 Y. Kamihara, T. Watanabe, M. Hirano, and H. Hosono, J. Am. Chem. Soc. 130, 3296 (2008).

2 C. de la Cruz, Q. Huang, J. W. Lynn, J. Li, W. R. II, J. L. Zarestky, H. A. Mook, G. F. Chen, J. L. Luo, N. L. Wang, et al., Nature (London) 453, 899 (2008).

3 S. A. J. Kimber, D. N. Argyriou, F. Yokaichiya, K. Habicht, S. Gerischer, T. Hansen, T. Chatterji, R. Klingeler, C. Hess, G. Behr, et al., Phys. Rev. B 78, 140503(R) (2008).

4 J. Zhao, Q. Huang, C. de la Cruz, S. Li, J. W. Lynn, Y. Chen, M. A. Green, G. F. Chen, G. Li, Z. Li, et al., Nature Mater. 7, 953 (2008).

5 Z.-A. Ren, W. Lu, J. Yang, W. Yi, X.-L. Shen, Z.-C. Li, G.-C. Che, X.-L. Dong, L.-L. Sun, F. Zhou, et al., Chin. Phys. Lett. 25, 2215 (2008).

${ }^{6}$ H. Kito, H. Eisaki, and A. Iyo, J. Phys. Soc. Jpn. 77, 063707 (2008).

7 G. Wu, Y. L. Xie, H. Chen, M. Zhong, R. H. Liu, B. C. Shi, Q. J. Li, X. F. Wang, T. Wu, Y. J. Yan, et al., J. Phys.: Condens. Matter 21, 142203 (2009).

${ }^{8}$ M. Rotter, M. Tegel, and D. Johrendt, Phys. Rev. Lett. 101, 107006 (2008).

${ }^{9}$ K. Sasmal, B. Lv, B. Lorenz, A. M. Guloy, F. Chen, Y.Y. Xue, and C.-W. Chu, Phys. Rev. Lett. 101, 107007 (2008).

10 X. C. Wang, Q. Q. Liu, Y. X. Lv, W. B. Gao, L. X. Yang, R. C. Yu, F. Y. Li, and C. Q. Jin, Solid State Communications 148, 538 (2008).

11 D. R. Parker, M. J. Pitcher, P. J. Baker, I. Franke, T. Lancaster, S. J. Blundell, and S. J. Clarke, Chemical Communications 2009, 2189 (2009).

12 F.-C. Hsu, J.-Y. Luo, K.-W. Yeh, T.-K. Chen, T.-W. Huang, P. M. Wu, Y.-C. Lee, Y.-L. Huang, Y.-Y. Chu, D.-C. Yan, et al., Proceedings of the National Academy of Sciences of the United States of America 105, 14262 (2008).

13 Y. Mizuguchi, F. Tomioka, S. Tsuda, T. Yamaguchi, and Y. Takano, Appl. Phys. Lett. 93, 152505 (2008).

${ }^{14}$ H. Ogino, Y. Matsumura, Y. Katsura, K. Ushiyama, S. Horii, K. Kishio, and J. Shimoyama, Superconductor Science and Technology 22, 075008 (2009).

15 X. Zhu, F. Han, G. Mu, P. Cheng, B. Shen, B. Zeng, and H.-H. Wen, Phys. Rev. B 79, 220512(R) (2009).

16 N. Kawaguchi, H. Ogino, Y. Shimizu, K. Kishio, and J. Shimoyama, Applied Physics Express 3, 063102 (2010).

17 H. Ogino, S. Sato, K. Kishio, J. Shimoyama, T. Tohei, and Y. Ikuhara, Appl. Phys. Lett. 97, 072506 (2010).

18 H. Ogino, K. Machida, A. Yamamoto, K. Kishio, J. Shimoyama, T. Tohei, and Y. Ikuhara, Superconductor Science and Technology 23, 115005 (2010).

19 P. M. Shirage, K. Kihou, C.-H. Lee, H. Kito, H. Eisaki, and A. Iyo, Appl. Phys. Lett. 97, 172506 (2010).

20 C.-H. Lee, A. Iyo, H. Eisaki, H. Kito, M. T. FernandezDiaz, T. Ito, K. Kihou, H. Matsuhata, M. Braden, and K. Yamada, J. Phys. Soc. Jpn. 77, 083704 (2008).

21 M. J. Han, Q. Yin, W. E. Pickett, and S. Y. Savrasov, Phys. Rev. Lett. 102, 107003 (2009).

22 K. Kuroki, H. Usui, S. Onari, R. Arita, and H. Aoki, Phys. Rev. B 79, 224511 (2009).

23 K. Horigane, H. Hiraka, and K. Ohyama, J. Phys. Soc. Jpn. 78, 074718 (2009).
${ }^{24}$ C. de la Cruz, W. Z. Hu, S. Li, Q. Huang, J. W. Lynn, M. A. Green, G. F. Chen, N. L. Wang, H. A. Mook, Q. Si, et al., Phys. Rev. Lett. 104, 017204 (2010).

25 T. Nomura, Y. Inoue, S. Matsuishi, M. Hirano, J. E. Kim, K. Kato, M. Takata, and H. Hosono, Superconductor Science and Technology 22, 055016 (2009).

26 H. Takahashi, K. Igawa, K. Arii, Y. Kamihara, M. Hirano, and H. Hosono, Nature (London) 453, 376 (2008).

27 S. Medvedev, T. M. McQueen, I. A. Troyan, T. Palasyuk, M. I. Eremets, R. J. Cava, S. Naghavi, F. Casper, V. Ksenofontov, G. Wortmann, et al., Nature Mater. 8, 630 (2009).

28 D. J. Singh and M.-H. Du, Phys. Rev. Lett. 100, 237003 (2008).

29 L. Boeri, O. V. Dolgov, and A. A. Golubov, Phys. Rev. Lett. 101, 026403 (2008).

${ }^{30}$ K. Haule, J. H. Shim, and G. Kotliar, Phys. Rev. Lett. 100, 226402 (2008).

31 I. I. Mazin, M. D. Johannes, L. Boeri, K. Koepernik, and D. J. Singh, Phys. Rev. B 78, 085104 (2008).

32 T. Yildirim, Phys. Rev. Lett. 102, 037003 (2009).

33 L. Boeri, M. Calandra, I. I. Mazin, O. V. Dolgov, and F. Mauri, Phys. Rev. B 82, 020506(R) (2010).

${ }^{34}$ F. Yndurain, Europhys. Lett. 94, 37001 (2011).

35 Y. Qiu, M. Kofu, W. Bao, S.-H. Lee, Q. Huang, T. Yildirim, J. R. D. Copley, J. W. Lynn, T. Wu, G. Wu, et al., Phys. Rev. B 78, 052508 (2008).

36 A. D. Christianson, M. D. Lumsden, O. Delaire, M. B. Stone, D. L. Abernathy, M. A. McGuire, A. S. Sefat, R. Jin, B. C. Sales, D. Mandrus, et al., Phys. Rev. Lett. 101, 157004 (2008).

37 T. Fukuda, A. Q. R. Baron, S. Shamoto, M. Ishikado, H. Nakamura, M. Machida, H. Uchiyama, S. Tsutsui, A. Iyo, H. Kito, et al., J. Phys. Soc. Jpn. 77, 103715 (2008).

38 M. L. Tacon, M. Krisch, A. Bosak, J.-W. G. Bos, and S. Margadonna, Phys. Rev. B 78, 140505(R) (2008).

39 R. Mittal, Y. Su, S. Rols, T. Chatterji, S. L. Chaplot, H. Schober, M. Rotter, D. Johrendt, and T. Brueckel, Phys. Rev. B 78, 104514 (2008).

40 R. Mittal, Y. Su, S. Rols, M. Tegel, S. L. Chaplot, H. Schober, T. Chatterji, D. Johrendt, and T. Brueckel, Phys. Rev. B 78, 224518 (2008).

41 R. Mittal, S. Rols, M. Zbiri, Y. Su, H. Schober, S. L. Chaplot, M. Johnson, M. Tegel, T. Chatterji, S. Matsuishi, et al., Phys. Rev. B 79, 144516 (2009).

42 R. Mittal, L. Pintschovius, D. Lamago, R. Heid, K.-P. Bohnen, D. Reznik, S. L. Chaplot, Y. Su, N. Kumar, S. K. Dhar, et al., Phys. Rev. Lett. 102, 217001 (2009).

43 D. Phelan, J. N. Millican, E. L. Thomas, J. B. Leão, Y. Qiu, and R. Paul, Phys. Rev. B 79, 014519 (2009).

${ }^{4}$ C.-H. Lee, K. Kihou, K. Horigane, S. Tsutsui, T. Fukuda, H. Eisaki, A. Iyo, H. Yamaguchi, A. Q. R. Baron, M. Braden, et al., J. Phys. Soc. Jpn. 79, 014714 (2010).

45 D. Reznik, K. Lokshin, D. C. Mitchell, D. Parshall, W. Dmowski, D. Lamago, R. Heid, K.-P. Bohnen, A. S. Sefat, M. A. McGuire, et al., arXiv: 0810.4941.

46 R. Mittal, M. Zbiri, S. Rols, Y. Su, Y. Xiao, H. Schober, S. L. Chaplot, M. Johnson, T. Chatterji, S. Matsuishi, et al., Phys. Rev. B 79, 214514 (2009).

47 S. E. Hahn, Y. Lee, N. Ni, P. C. Canfield, A. I. Goldman, 
R. J. McQueeney, B. N. Harmon, A. Alatas, B. M. Leu, E. E. Alp, et al., Phys. Rev. B 79, 220511(R) (2009).

48 D. Reznik, K. Lokshin, D. C. Mitchell, D. Parshall, W. Dmowski, D. Lamago, R. Heid, K.-P. Bohnen, A. S. Sefat, M. A. McGuire, et al., Phys. Rev. B 80, 214534 (2009).

49 M. L. Tacon, T. R. Forrest, C. Rüegg, A. Bosak, A. C. Walters, R. Mittal, H. M. Rønnow, N. D. Zhigadlo, S. Katrych, J. Karpinski, et al., Phys. Rev. B 80, 220504(R) (2009).

50 M. L. Tacon, T. R. Forrest, C. Rüegg, A. Bosak, J. Noffsinger, A. C. Walters, P. Toulemonde, A. Palenzona, N. D. Zhigadlo, J. Karpinski, et al., arXiv: 1005.4165.

51 M. Zbiri, R. Mittal, S. Rols, Y. Su, Y. Xiao, H. Schober, S. L. Chaplot, M. R. Johnson, T. Chatterji, Y. Inoue, et al., J. Phys.: Condens. Matter 22, 315701 (2010).

52 M. Ishikado, S. Shamoto, H. Kito, A. Iyo, H. Eisaki, T. Ito, and Y. Tomioka, Physica C 469, 901 (2009).

53 C. Hess, A. Kondrat, A. Narduzzo, J. E. Hamann-Borrero, R. Klingeler, J. Werner, G. Behr, and B. Büchner, Europhys. Lett. 87, 17005 (2009).

54 M. Rotter, M. Tegel, D. Johrendt, I. Schellenberg, W. Hermes, and R. Pöttgen, Phys. Rev. B 78, 020503(R) (2008).

55 J. Zhao, Q. Huang, C. de la Cruz, J. W. Lynn, M. D. Lumsden, Z. A. Ren, J. Yang, X. Shen, X. Dong, Z. Zhao, et al., Phys. Rev. B 78, 132504 (2008).

56 A. Q. R. Baron, Y. Tanaka, S. Goto, K. Takeshita, T. Matsushita, and T. Ishikawa, J. Phys. Chem. Solids 61, 461 (2000).

57 A. Q. R. Baron, J. P. Sutter, S. Tsutsui, H. Uchiyama, T. Masui, S. Tajima, R. Heid, and K.-P. Bohnen, J. Phys. Chem. Solids 69, 3100 (2008).

58 G. Kresse and J. Hafner, Phys. Rev. B 47, (R)558 (1993).

59 G. Kresse and J. Furthmüller, Computational Materials Science 6, 15 (1996).

60 G. Kresse and J. Furthmüller, Phys. Rev. B 54, 11169 (1996).

61 P. E. Blöchl, Phys. Rev. B 50, 17953 (1994).

${ }^{62}$ G. Kresse and D. Joubert, Phys. Rev. B 59, 1758 (1999).

63 K. Parlinski, Z. Q. Li, and Y. Kawazoe, Phys. Rev. Lett. 78, 4063 (1997).

64 S. Ishibashi, K. Terakura, and H. Hosono, J. Phys. Soc. Jpn. 77, 053709 (2008).

65 J. Zhao, D.-X. Yao, S. Li, T. Hong, Y. Chen, S. Chang, W. R. II, J. W. Lynn, H. A. Mook, G. F. Chen, et al., Phys. Rev. Lett. 101, 167203 (2008).

66 J. Zhao, D. T. Adroja, D.-X. Yao, R. Bewley, S. Li, X. F. Wang, G. Wu, X. H. Chen, J. Hu, and P. Dai, Nature Physics 5, 555 (2009).

67 S. Higashitaniguchi, M. Seto, S. Kitao, Y. Kobayashi, M. Saito, R. Masuda, T. Mitsui, Y. Yoda, Y. Kamihara, M. Hirano, et al., Phys. Rev. B 78, 174507 (2008).

68 S. Tsutsui, C.-H. Lee, C. Tassel, Y. Yoshida, Y. Yoda, K. Kihou, A. Iyo, and H. Eisaki, J. Phys. Soc. Jpn. 79, 013706 (2010).

69 O. Delaire, M. S. Lucas, A. M. dos Santos, A. Subedi, A. S. Sefat, M. A. McGuire, L. Mauger, J. A. Mun̄oz, C. A. Tulk, Y. Xiao, et al., Phys. Rev. B 81, 094504 (2010).

70 V. Ksenofontov, G. Wortmann, A. I. Chumakov, T. Gasi, S. Medvedev, T. M. McQueen, R. J. Cava, and C. Felser, Phys. Rev. B 81, 184510 (2010).

71 A. P. Litvinchuk, V. G. Hadjiev, M. N. Iliev, B. Lv,
A. M. Guloy, and C. W. Chu, Phys. Rev. B 78, 060503(R) (2008).

72 S. C. Zhao, D. Hou, Y. Wu, T. L. Xia, A. M. Zhang, G. F. Chen, J. L. Luo, N. L. Wang, J. H. Wei, Z. Y. Lu, et al., Supercond. Sci. Technol. 22, 015017 (2009).

73 Y. Gallais, A. Sacuto, M. Cazayous, P. Cheng, L. Fang, and H. H. Wen, Phys. Rev. B 78, 132509 (2008).

74 L. Zhang, T. Fujita, F. Chen, D. L. Feng, S. Maekawa, and M. W. Chen, Phys. Rev. B 79, 052507 (2009).

75 M. Rahlenbeck, G. L. Sun, D. L. Sun, C. T. Lin, B. Keimer, and C. Ulrich, Phys. Rev. B 80, 064509 (2009).

76 L. Chauvière, Y. Gallais, M. Cazayous, A. Sacuto, M. A. Méasson, D. Colson, and A. Forget, Phys. Rev. B 80, 094504 (2009).

77 K.-Y. Choi, D. Wulferding, P. Lemmens, N. Ni, S. L. Bud'ko, and P. C. Canfield, Phys. Rev. B 78, 212503 (2008).

78 K.-Y. Choi, P. Lemmens, I. Eremin, G. Zwicknagl, H. Berger, G. L. Sun, D. L. Sun, and C. T. Lin, J. Phys.: Condens. Matter 22, 115802 (2010).

79 M. Granath, J. Bielecki, J. Holmlund, and L. Börjesson, Phys. Rev. B 79, 235103 (2009).

${ }^{80}$ P. Kumar, A. Kumar, S. Saha, D. V. S. Muthu, J. Prakash, S. Patnaik, U. V. Waghmare, A. K. Ganguli, and A. K. Sood, Solid State Communications 150, 557 (2010).

81 T.-L. Xia, D. Hou, S. C. Zhao, A. M. Zhang, G. F. Chen, J. L. Luo, N. L. Wang, J. H. Wei, Z.-Y. Lu, and Q. M. Zhang, Phys. Rev. B 79, 140510(R) (2009).

82 K. Okazaki, S. Sugai, S. Niitaka, and H. Takagi, Phys. Rev. B 83, 035103 (2011).

83 A. M. Zhang, J. H. Xiao, J. B. He, D. M. Wang, G. F. Chen, and Q. M. Zhang, arXiv: 1101.2168.

${ }^{84}$ S. Sugai, Y. Mizuno, K. Kiho, M. Nakajima, C. H. Lee, A. Iyo, H. Eisaki, and S. Uchida, Phys. Rev. B 82, 140504(R) (2010).

85 S. I. Mirzaei, V. Guritanu, A. B. Kuzmenko, C. Senatore, D. van der Marel, G. Wu, R. H. Liu, and X. H. Chen, arXiv: 0806.2303 .

${ }^{86}$ A. Akrap, J. J. Tu, L. J. Li, G. H. Cao, Z. A. Xu, and C. C. Homes, Phys. Rev. B 80, 180502(R) (2009).

87 D. Wu, N. Barišić, N. Drichko, S. Kaiser, A. Faridian, M. Dressel, S. Jiang, Z. Ren, L. J. Li, G. H. Cao, et al., Phys. Rev. B 79, 155103 (2009).

88 E. van Heumen, Y. Huang, S. de Jong, A. B. Kuzmenko, M. S. Golden, and D. van der Marel, Europhys. Lett. 90, 37005 (2010).

89 B. Mansart, D. Boschetto, A. Savoia, F. Rullier-Albenque, A. Forget, D. Colson, A. Rousse, and M. Marsi, Phys. Rev. B 80, 172504 (2009).

90 T. Mertelj, P. Kusar, V. V. Kabanov, L. Stojchevska, N. D. Zhigadlo, S. Katrych, Z. Bukowski, J. Karpinski, S. Weyeneth, and D. Mihailovic, Phys. Rev. B 81, 224504 (2010).

91 H. Takahashi, Y. Kamihara, H. Koguchi, T. Atou, H. Hosono, I. Katayama, J. Takeda, M. Kitajima, and K. G. Nakamura, J. Phys. Soc. Jpn. 80, 013707 (2011).

92 M. Zbiri, H. Schober, M. R. Johnson, S. Rols, R. Mittal, Y. Su, M. Rotter, and D. Johrendt, Phys. Rev. B 79, 064511 (2009).

93 T. Yildirim, Physica C 469, 425 (2009).

94 S. O. Diallo, V. P. Antropov, T. G. Perring, C. Broholm, J. J. Pulikkotil, N. Ni, S. L. Bud'ko, P. C. Canfield, A. Kreyssig, A. I. Goldman, et al., Phys. Rev. Lett. 102, 
187206 (2009).

95 S.-H. Lee, G. Xu, W. Ku, J. S. Wen, C. C. Lee, N. Katayama, Z. J. Xu, S. Ji, Z. W. Lin, G. D. Gu, et al., Phys. Rev. B 81, 220502(R) (2010).

96 T. Shimojima, K. Ishizaka, Y. Ishida, N. Katayama, K. Ohgushi, T. Kiss, M. Okawa, T. Togashi, X.-Y. Wang, C.-T. Chen, et al., Phys. Rev. Lett. 104, 057002 (2010).

97 W. Lv, F. Krüger, and P. Phillips, Phys. Rev. B 82, 045125 (2010).

98 J.-H. Chu, J. G. Analytis, K. D. Greve, P. L. McMahon, Z. Islam, Y. Yamamoto, and I. R. Fisher, Science 329, $824(2010)$.

99 I. I. Mazin and M. D. Johannes, Nature Physics 5, 141 (2009).

100 S. Kitao, Y. Kobayashi, S. Higashitaniguchi, M. Saito, Y. Kamihara, M. Hirano, T. Mitsui, H. Hosono, and M. Seto, J. Phys. Soc. Jpn. 77, 103706 (2008).

101 H. Luetkens, H.-H. Klauss, R. Khasanov, A. Amato, R. Klingeler, I. Hellmann, N. Leps, A. Kondrat, C. Hess, A. Köhler, et al., Phys. Rev. Lett. 101, 097009 (2008).

102 J. P. Carlo, Y. J. Uemura, T. Goko, G. J. MacDougall, J. A. Rodriguez, W. Yu, G. M. Luke, P. Dai, N. Shannon,
S. Miyasaka, et al., Phys. Rev. Lett. 102, 087001 (2009).

103 S. Takeshita, R. Kadono, M. Hiraishi, M. Miyazaki, A. Koda, S. Matsuishi, and H. Hosono, Phys. Rev. Lett. 103, 027002 (2009).

104 K. Ohishi, Y. Ishii, K. Miyazawa, H. Fukazawa, I. Watanabe, Y. Kohori, P. M. Shirage, H. Kito, A. Iyo, and H. Eisaki, J. Phys. Soc. Jpn. 80, 024703 (2011).

105 F. Bondino, E. Magnano, M. Malvestuto, F. Parmigiani, M. A. McGuire, A. S. Sefat, B. C. Sales, R. Jin, D. Mandrus, E. W. Plummer, et al., Phys. Rev. Lett. 101, 267001 (2008).

106 A. D. Christianson, E. A. Goremychkin, R. Osborn, S. Rosenkranz, M. D. Lumsden, C. D. Malliakas, I. S. Todorov, H. Claus, D. Y. Chung, M. G. Kanatzidis, et al., Nature (London) 456, 930 (2008).

107 M. Ishikado, R. Kajimoto, S. Shamoto, M. Arai, A. Iyo, K. Miyazawa, P. M. Shirage, H. Kito, H. Eisaki, S. Kim, et al., J. Phys. Soc. Jpn. 78, 043705 (2009).

108 S. Wakimoto, K. Kodama, M. Ishikado, M. Matsuda, R. Kajimoto, M. Arai, K. Kakurai, F. Esaka, A. Iyo, H. Kito, et al., J. Phys. Soc. Jpn. 79, 074715 (2010). 